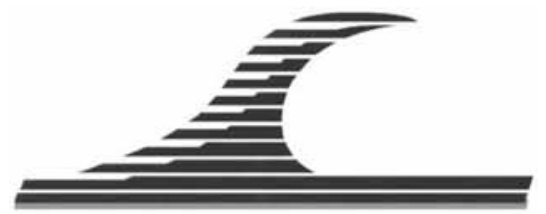

Revue Paralia, Volume 4 (2011) pp 1.1-1.14

Mots-clés : Baie, Estuaire, Morphobathymétrie,

Hydrodynamique, Lagune Ebrié, Côte d'Ivoire

(c) Editions Paralia CFL

\title{
Hydrodynamique de l'estuaire de la lagune Ebrié (Côte d'Ivoire)
}

\author{
Sylvain MONDE ${ }^{1}$, Aoua Sougo COULIBALY ${ }^{1}$, \\ Ted-Edgard WANGO ${ }^{1}$, Kouamé AKA ${ }^{1}$
}

1. Université de Cocody, Unité de Formation et de Recherches des Sciences de la Terre et des Ressources Minières, Département de géosciences marines, 22 B.P. 582 Abidjan 22, Côte d'Ivoire. sylvain.monde@univ-cocody.ci

\section{Résumé :}

Les baies estuariennes d'Abidjan subissent une forte pression anthropique qui modifie la circulation des masses d'eaux. En outre, ces baies présentent des signes de pollution. Afin de mieux cerner les processus qui s'y déroulent des approches morphobathymétrique et dynamique ont été mise en œuvre. Il en ressort que les baies estuariennes présentent des morphologies symétriques par rapport à l'axe central de la lagune Ebrié. Dans les chenaux étroits, les courants sont exclusivement alternatifs. Les vitesses des courants dans les baies de Cocody et de Biétri sont décrites. Les évolutions du niveau de l'eau, de la salinité et de la température sont étudiées dans la lagune sur 200 jours. Les variations de salinité dans la lagune s’agencent dans les saisons continentale et marine et dépendent des fortes précipitations et des crues fluviales. Il en est de même pour l'évolution de la température des eaux qui varie en fonction des précipitations et des débits fluviaux.

Soumis le 6 avril 2010, accepté le 10 janvier 2011, en ligne le 28 février 2011.

La seule version examinée est celle écrite en français. La ou les autres versions n'étant pas examinées par le comité de rédaction de la revue, sont donc publiées sous l'entière responsabilité du ou des auteurs. 


\section{Introduction}

Dans son acception la plus large un estuaire est un plan d'eau côtier partiellement confiné ayant une connexion libre avec l'océan et dans lequel l'eau marine est diluée de façon mesurable par l'eau douce dérivée des ruissellements continentaux (PRITCHARD, 1967). C'est donc le milieu privilégié de transition entre les domaines continentaux et océaniques. PERILLO (1995) complète cette définition par l'emploi des limites de l'action dynamique de la marée en amont, en précisant que l'eau marine peut pénétrer par un ou plusieurs bras ouverts sur le domaine marin. Ces estuaires et zones littorales représentent un intérêt scientifique croissant reposant sur leur importance socio-économique manifeste alors que près de $60 \%$ de la population mondiale vit à moins de $60 \mathrm{~km}$ des côtes.

En Afrique de l'Ouest, l'estuaire de la lagune Ebrié (en Côte d'Ivoire) est séparé de l'océan Atlantique par un cordon sableux, traversé artificiellement, depuis 1950, par le canal de Vridi. Les baies de cet estuaire constituent un milieu relativement renouvelé depuis l'ouverture de ce canal (figure 1). Les apports d'eaux continentales sont constitués pour les deux tiers de ceux du fleuve Comoé. Il débouche à l'extrémité orientale de la lagune Ebrié et en raison du volume de sa crue, contribue à une forte variabilité interannuelle (DURAND \& GUIRAL, 1994).

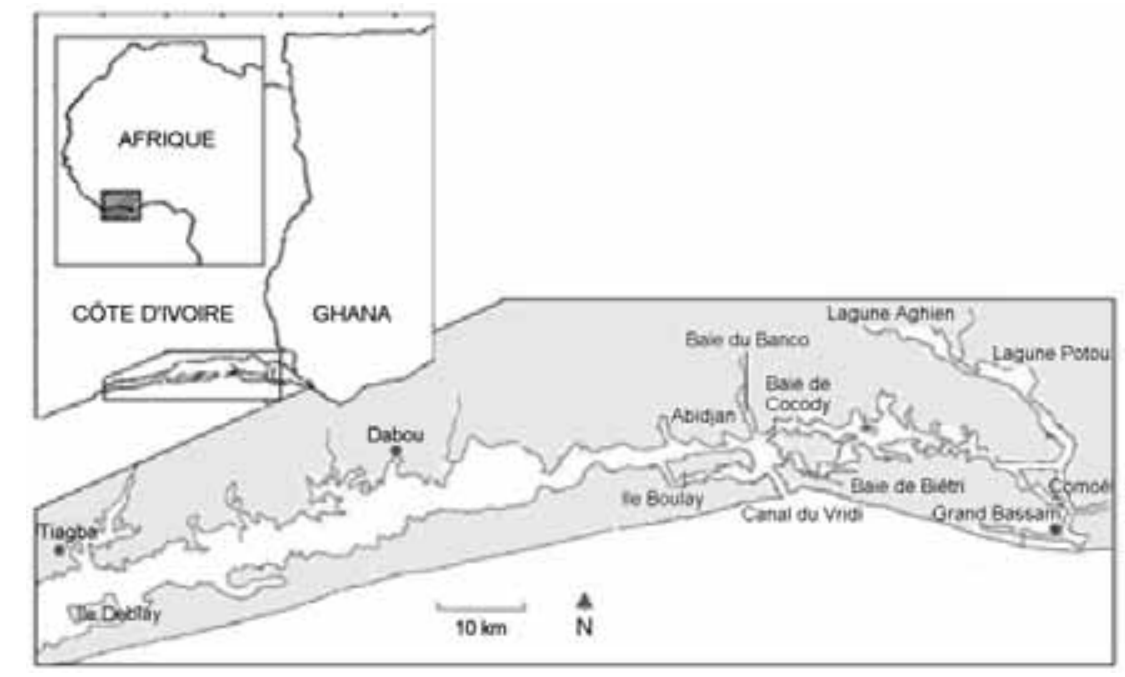

Figure 1. La lagune Ebrié et les baies estuariennes d'Abidjan.

Les baies estuariennes d'Abidjan subissent une pression du fait des activités humaines sans cesse croissantes depuis le siècle dernier. Ces environnements confinés constituent les points principaux d'aboutissement du réseau d'égouts de la ville d'Abidjan. Parallèlement à son évolution démographique, l'agglomération abidjanaise connaît un essor industriel d'une importance notable. En outre, le développement des installations portuaires induit, par l'extension du trafic maritime, d'inévitables pressions sur l'environnement. Il y a également la construction du réseau routier qui nécessite la 
création de buses, de digues comme au niveau des baies de Biétri et de Koumassi (figure 2). Cet anthropisme aura indéniablement un impact sur la circulation des masses d'eau dans l'estuaire. La présente note analyse la réponse de cet estuaire face aux forçages naturels et anthropiques. Ces approches morphobathymétriques et hydrodynamiques de l'estuaire de la lagune Ebrié s'attachent à l'étude des masses d'eau dans ce système lagunaire et à la caractérisation des échanges avec les environnements limitrophes.

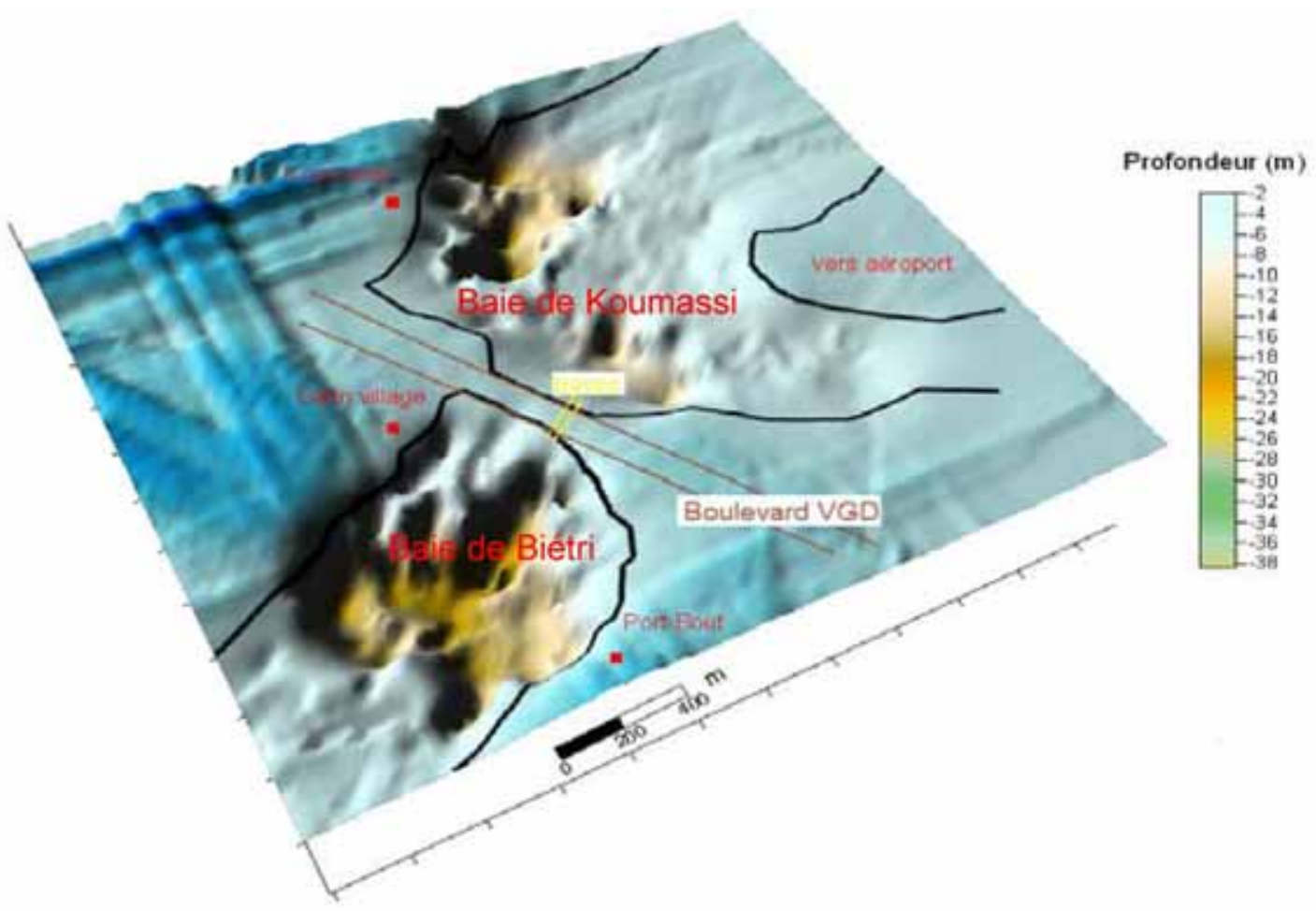

Figure 2. MNT des baies de Bietri et Koumassi.

\section{Méthodologie}

Les données XYZ (longitude, latitude et profondeur) des minutes d'échosondage (PAA, 1999) sont exportées dans un logiciel de cartographie (Surfer). Ce dernier génère des cartes bathymétriques (2D).

Un courantomètre à Effet Doppler de type DCM12 (Aanderaa) a été utilisé pour déterminer la vitesse d'écoulement dans la colonne d'eau à différentes profondeurs pour un site donné et durant un cycle de marée. Ce courantomètre donne, en outre, la variation du niveau de l'eau en temps réel et la direction du courant. Les vitesses de courant mesurées pendant le flot sont comptées positivement. Par contre, les vitesses sont comptées négativement pendant le jusant. 
L'utilisation du sondeur OBS-3A pour l'acquisition de données hydrodynamiques en continu pendant 20 mois, s'est faite dans le port d'Abidjan. Il est équipé de capteurs dont le seuillage a été prédéfini (tableau 1) en fonction de l'hydrologie de la lagune Ebrié. Le pas de temps de la mesure est de 300 secondes. Cela représente 4320 mesures pour 15 jours (correspondant à l'autonomie de la batterie d'alimentation du sondeur). Ce qui reste inférieur à la quantité réelle de données que le sondeur peut enregistrer, en alimentation continue, soit 6640 mesures. Néanmoins, ce pas de temps permet d'acquérir 65\% des données soit les 4320 mesures. C’est un équilibre entre la représentativité et la quantité d'échantillon à traiter. En outre, les moyennes journalières et bimensuelles du niveau d'eau, de la salinité, de la température, ont été déterminées par la méthode arithmétique classique. Pour ce calcul, un pas d'échantillonnage de 1 mesure tous les 10 min a été pris.

Tableau 1. Paramètres de configuration et degré d'incertitude des capteurs OBS-3A.

\begin{tabular}{lll}
\hline Capteurs & Seuils & Incertitudes \\
\hline Turbidité & $N T U$ & $2 \%$ \\
Température & $20^{\circ} \mathrm{C}$ & $\pm 0,0075^{\circ} \mathrm{C}$ \\
Pression & $d B a r s$ & $\pm 0,2 \%$ \\
Salinité & $0 \mathrm{PSU}$ & $\pm 0,1 \mathrm{PSU}$ \\
\hline
\end{tabular}

\section{Résultats et discussions}

\subsection{Carte bathymétrique de l'estuaire de la lagune Ebrié}

L'estuaire présente une profondeur moyenne de $5 \mathrm{~m}$, ce qui en fait un environnement peu profond. D’importantes structures s'observent dans le bassin central avec une morphologie particulière. Ces morphostructures (figure 3) s'observent à l'entrée du chenal Sud de l'île Boulay (M1) et près des quais (Sud et Nord) du port d'Abidjan (M2 et M3). Les morphostructures M2 (haut-fond) et M3 (dépression) ont une direction $\mathrm{N} 180^{\circ}$ et se localisent dans le chenal principal de navigation portuaire (tableau 2). En outre, les fortes pentes se localisent à proximité des quais et les plus faibles, dans le chenal de navigation (MONDE, 2004). Ce secteur est très souvent dragué afin de faciliter le trafic maritime. 


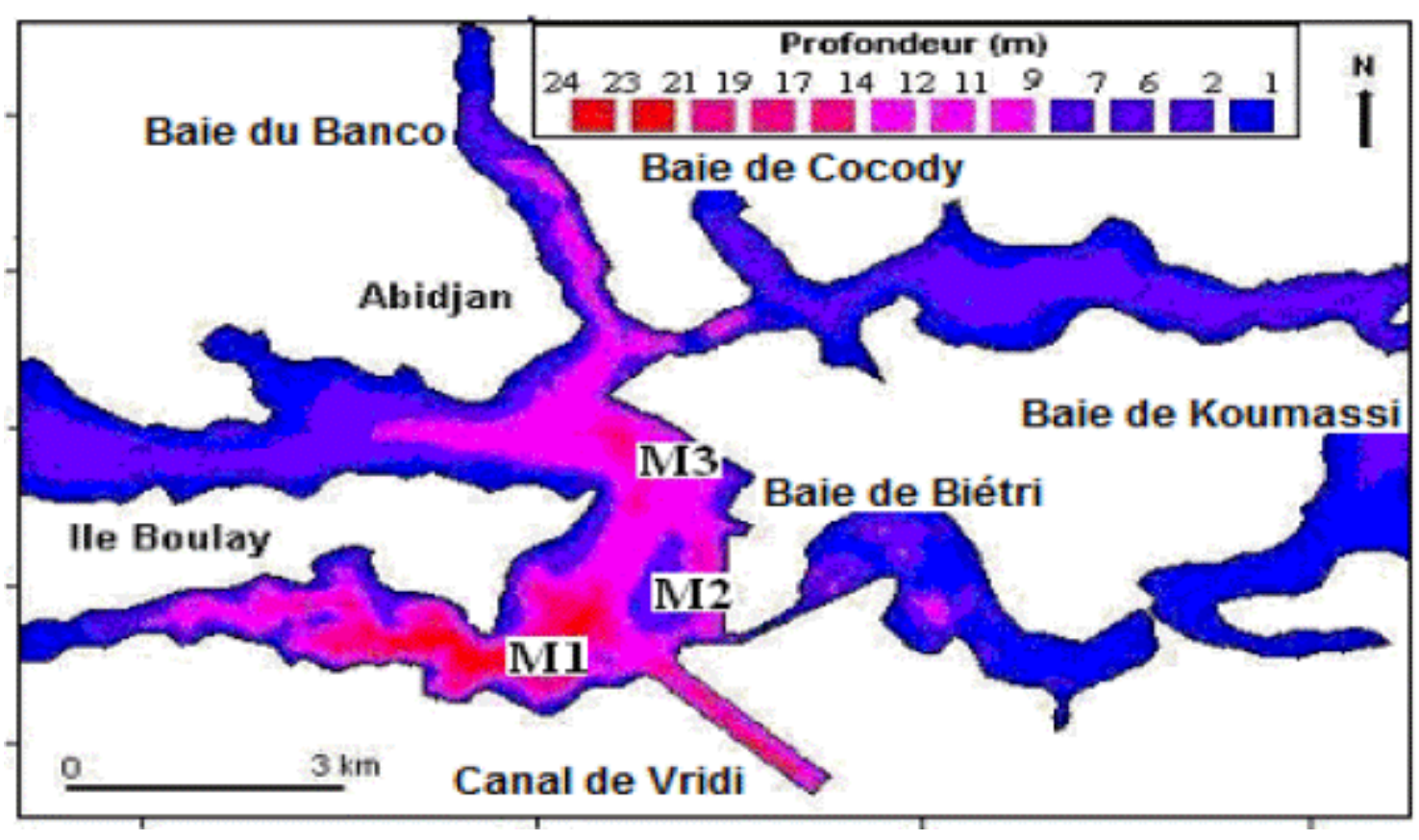

Figure 3. Morphostructures (M) dans les chenaux de la lagune Ebrié.

Tableau 2. Caractéristiques de quelques morphostructures de la lagune Ebrié.

\begin{tabular}{llll}
\hline & M1 & M2 & M3 \\
\hline Longueur (km) & 1,65 & 1,65 & 1,55 \\
Largeur (km) & 0,2 & 0,3 & $0,2-0,8$ \\
Profondeur (m) & 4,75 & 2,35 & 4,70 \\
\hline
\end{tabular}

3.2 Morphobathymétrie et circulation des masses d'eau dans les baies

L'analyse de la morphobathymétrie et de la circulation des masses d'eau portera sur deux baies d'Abidjan qui constituent non seulement le déversoir des rejets domestiques pour les habitants des quartiers précaires situés en bordure de la lagune Ebrié mais elles aussi, sont soumises à d'intenses activités industrielles (entreprises de carénages, de recyclage des métaux, de teinture, etc.).

\subsubsection{Baie de Cocody}

Le chenal central de la baie recoupe perpendiculairement le chenal principal de la lagune Ebrié. Les profondeurs sont faibles dans cette baie (0,5 à 3,5 m), sauf à la confluence avec la lagune, où l'on trouve une fosse de $-5 \mathrm{~m}$ (figure 4). L'analyse des pentes montre des zones de fortes pentes (3,5\%) et de faibles pentes (1,75\%). Cette dernière s’observe à la rive Sud. 


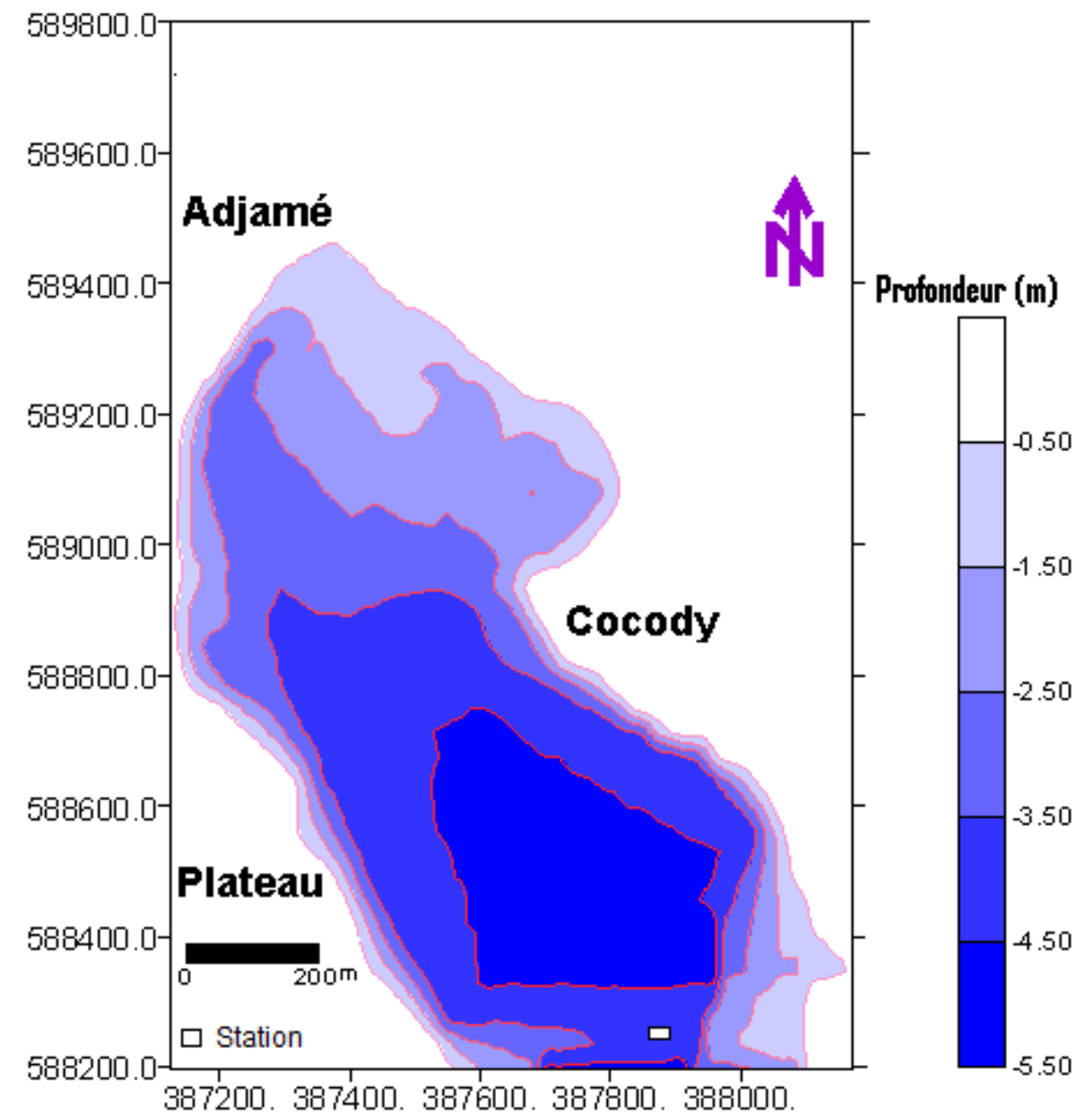

Figure 4. Carte bathymétrique de la baie de Cocody (projection UTM 30N).

La figure 5 présente la variation temporelle des vitesses de courants selon la profondeur. Le flot atteint son maximum dans la tranche d'eau superficielle avec une vitesse de $0,42 \mathrm{~m} \mathrm{~s}^{-1}$. En profondeur $(3,6 \mathrm{~m})$, la vitesse atteint $0,50 \mathrm{~m} \mathrm{~s}^{-1}$. Le jusant est maximum avec une vitesse de $0,72 \mathrm{~m} \mathrm{~s}^{-1}$ en surface et $0,68 \mathrm{~m} \mathrm{~s}^{-1}$ en profondeur. Le jusant et le flot ont la même durée (6 h). L'évolution des vitesses de courant de surface et de fond est similaire. Les vitesses restent inférieures à $1 \mathrm{~m} \mathrm{~s}^{-1}$. Toutefois, les courants de marée augmentent progressivement. En effet, ils varient de 0,1 à $0,5 \mathrm{~m} \mathrm{~s}^{-1}$ durant le flot (entre 0 et 3,66 $\mathrm{m}$ de profondeur) et de $-0,10$ à $-0,60 \mathrm{~m} \mathrm{~s}^{-1}$ lors du jusant. Ils atteignent un maximum à mi-période d'écoulement $\left(0,50 \mathrm{~m} \mathrm{~s}^{-1}\right.$ pour le courant de flot) et diminuent jusqu'au renversement $\left(-0,60 \mathrm{~m} \mathrm{~s}^{-1}\right.$ pour le courant de jusant). Ces variations s'observent de la surface $(0 \mathrm{~m})$ au fond des eaux (3,66 m).

Les directions du courant dans la baie de Cocody sont montrées sur la figure 6, Les courants sont presque alternatifs (direction ENE pendant le flot WSW en jusant) ce qui indiquerait la présence d'un chenal. 
Hydrodynamique de l'estuaire de la lagune Ebrié (Côte d'Ivoire) : 1.7

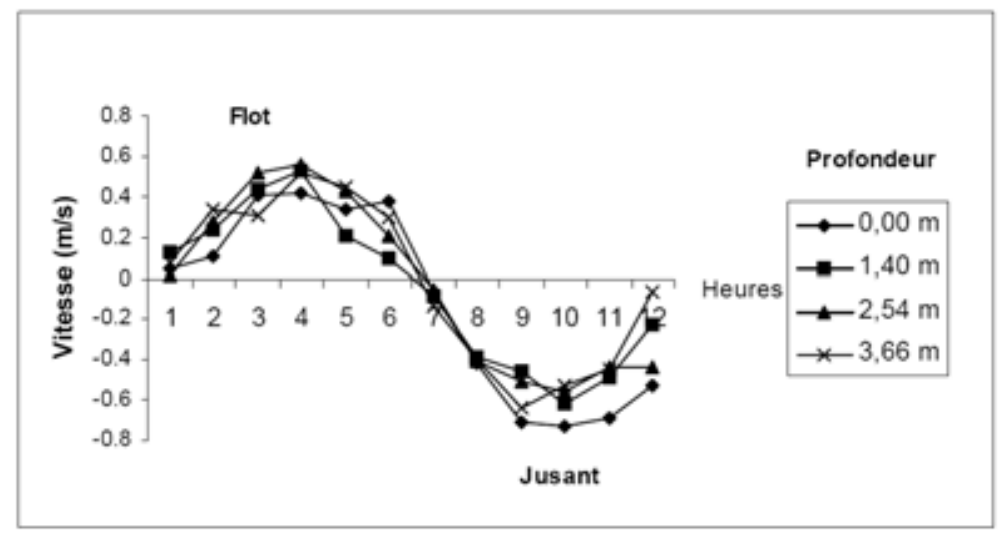

Figure 5. Évolution temporelle des vitesses de courant dans la baie de Cocody.

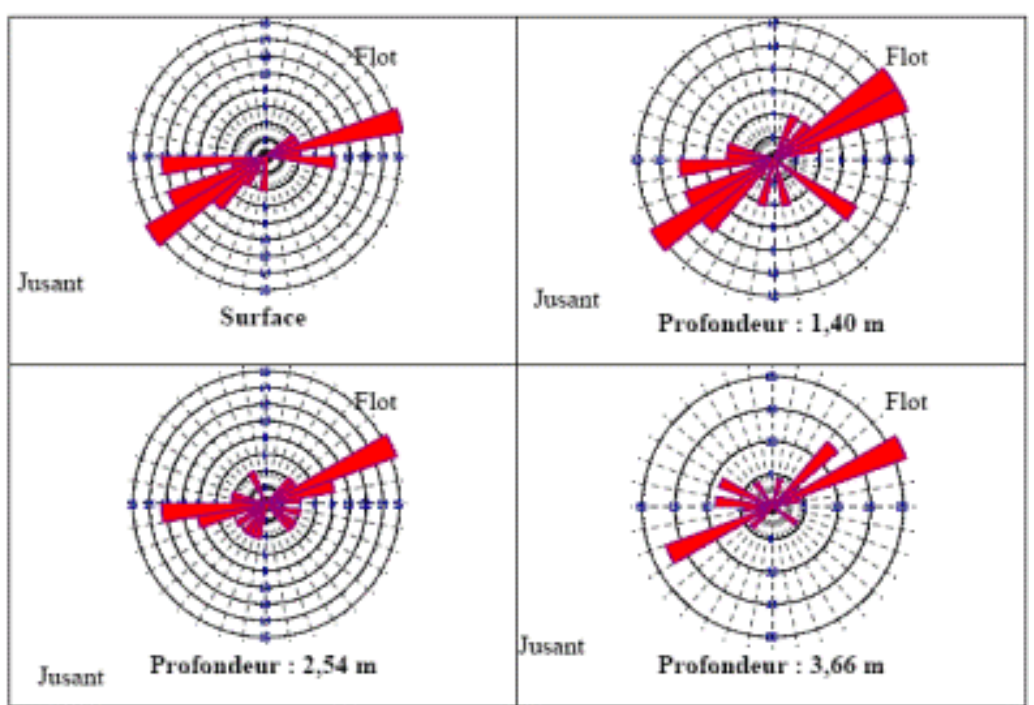

Figure 6. Directions des courants dans la baie de Cocody.

\subsubsection{Baie de Biétri}

Des chenaux se distinguent dans la baie. Le bras Sud, plus profond ( $>10 \mathrm{~m})$, rejoint celui situé au Nord qui a une allure curviligne. Des dépressions, de pente variant de 1,75 à 5\%, s'observent au fond de la baie. Elles s'expliqueraient par le dragage des fonds (figure 7).

Le flot a une vitesse maximale à -8,3 m de profondeur (figure 8). Le jusant dure $1 \mathrm{~h}$ de plus que le flot. Le maximum de jusant est observé en profondeur $(8,3 \mathrm{~m})$ avec une vitesse de $0,70 \mathrm{~m} \mathrm{~s}^{-1}$. On note une variation de vitesse entre les courants du fond et de surface. En profondeur, les courants sont tous giratoires (figure 9). 


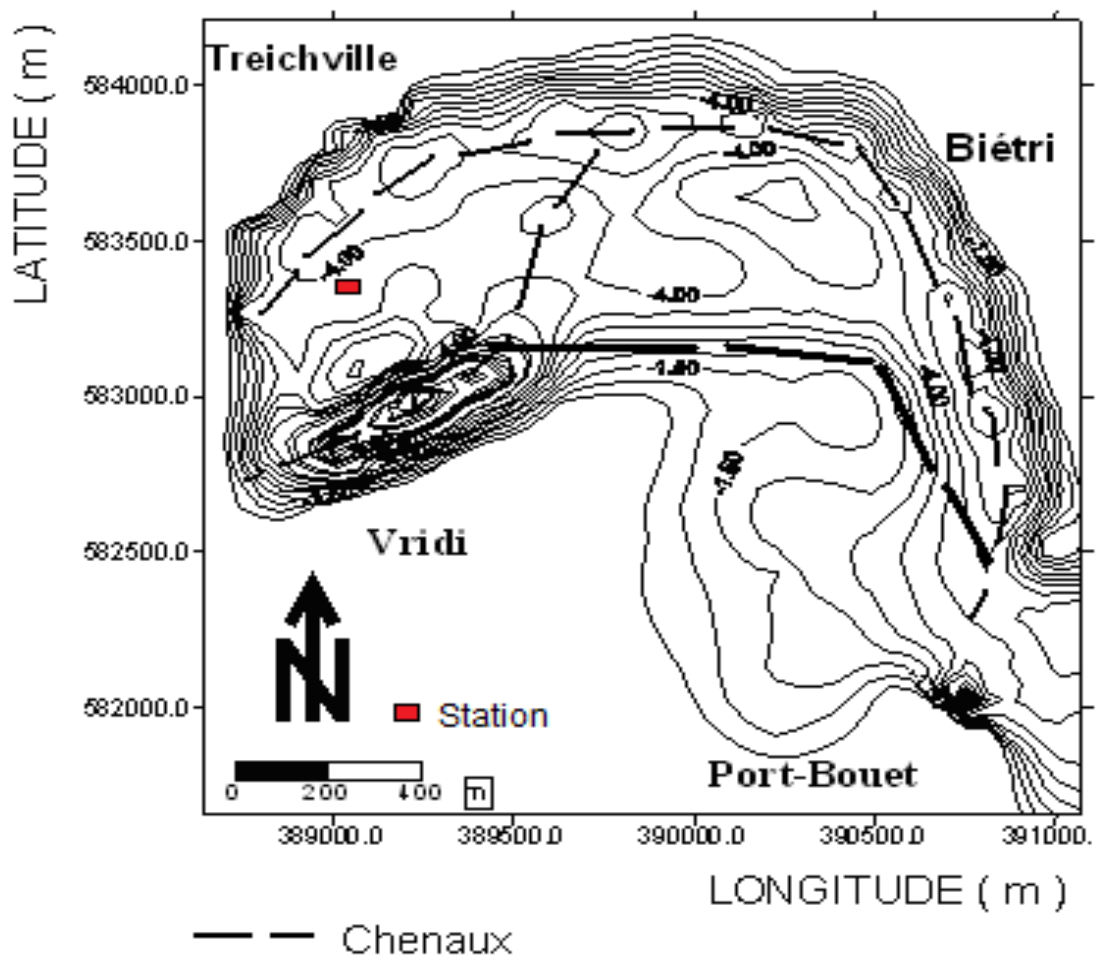

Figure 7. Carte bathymétrique la baie de Biétri (projection UTM 30N).

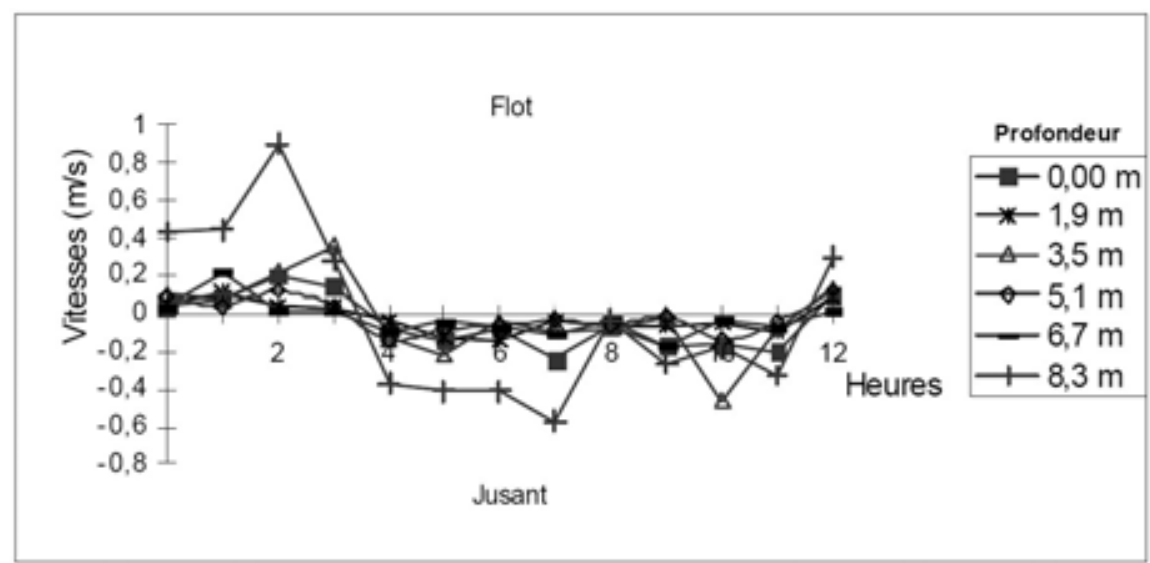

Figure 8. Vitesses de courant en vive-eau dans la baie de Biétri.

Des buses ont été crées entre les baies de Koumassi et de Biétri lors de la mise en place de la digue sur laquelle a été construit le boulevard VGE (reliant le centre ville d'Abidjan à la périphérie, voir figure 2). Les études courantologiques ante et post buses (LEMASSON et al. 1981; GUIRAL \& LANUSSE, 1984) ont permis d'apprécier l'évolution des courants. Elles montrent une répartition spatiale constante des courants. En outre, les courants croissent en intensité. Ils passent de 0,2 à $0,9 \mathrm{~m} \mathrm{~s}^{-1}$ et de 0 à $1,6 \mathrm{~m} \mathrm{~s}^{-1}$ respectivement dans la partie occidentale et orientale de la baie. Les courants à forte intensité se localisent à proximité du canal de Vridi et les plus faibles, près des 
buses. Dans les couches profondes les courants sont giratoires et les eaux sont mal renouvelées. Ces analyses concordent avec les travaux antérieurs (TASTET, 1979 ; GALLARDO, 1978) et montrent l'impact de la pression due aux activités humaines, dans l'évolution hydrodynamique de la baie de Biétri (figure 9).

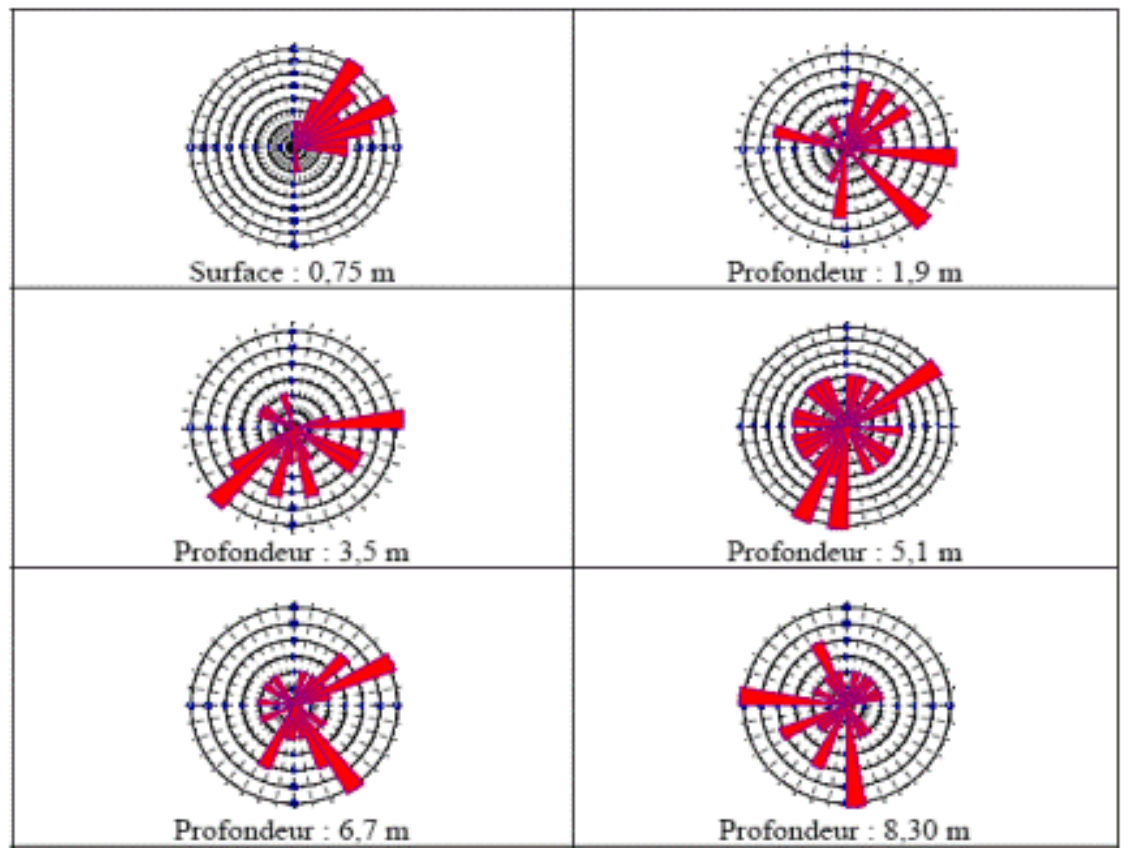

Figure 9. Fréquences des directions des courants dans la baie de Biétri.

\subsection{Hydrodynamique en lagune Ebrié}

\subsubsection{Marnage et hauteur d'eau}

L'analyse des variations de niveau d'eau sur 200 jours indique une moyenne de $+1,01 \mathrm{~m}$ par rapport à un référentiel local. Le marnage en vive eau varie de 0,55 à 0,80 m. En morte eau, le marnage est plus faible. Il varie de 0,30 à $0,35 \mathrm{~m}$. Le déchet dure 8 jours tandis que le revif est plus court, il dure 7 jours. La courbe d'évolution de la moyenne journalière de la hauteur d'eau en lagune Ebrié sur une période de 200 jours présente trois domaines (figure 10) :

- De février à avril ( $1^{\text {er }}-40^{\text {ème }}$ jour $)$, elle part d'un niveau relativement bas $(0,95 \mathrm{~m})$, passe par un maximum $(1,05 \mathrm{~m})$ et redescend à une faible hauteur $(0,90 \mathrm{~m})$ au bout du $40^{\text {ème }}$ jour. La dénivelée engendrée est de $0,10 \mathrm{~m}$.

- D'avril à juin, soit du $40^{\text {ème }}$ jour au $120^{\text {ème }}$ jour, la courbe est d'abord croissante, puis elle passe 0,90 à $1,09 \mathrm{~m}$ au bout du $65^{\text {ème }}$ jour. Elle se stabilise à cette hauteur jusqu'au $108^{\text {ème }}$ jour avant de décroître pour atteindre la valeur 1,00 $\mathrm{m}$. La dénivelée calculée est de $0,15 \mathrm{~m}$. 
- Le troisième domaine de la courbe part de la fin juin à août soit $120^{\text {ème }}$ jour au $200^{\text {ème }}$ jour. Dans cette partie de la courbe, on observe un niveau moyen qui passe de 1,05 à $1,07 \mathrm{~m}$ pour se stabiliser ensuite à $1,06 \mathrm{~m}$ au $160^{\text {ème }}$ jour.

Cette courbe montre une évolution irrégulière du niveau de la lagune. Les variations de

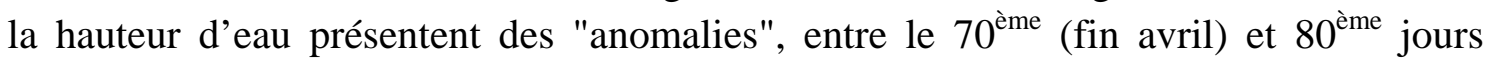
(début mai), dues à la surcote fluviale produite après les crues de l'Agnéby et La Mé (DURAND \& GUIRAL, 1994). De février à juin, la hausse progressive de la hauteur d'eau est due aux précipitations (période de transition entre les saisons de pluie continentale et lagunaire) et aux apports de La Mé et de l’Agnéby. De juin à août, la baisse de la hauteur du plan d'eau lagunaire est due à la rareté des précipitations. En effet, la petite saison sèche continentale débute à cette période et la saison de pluie lagunaire s’achève. La crue du Comoé permet une élévation des eaux lagunaires $(0,10$ à $0,50 \mathrm{~m})$.

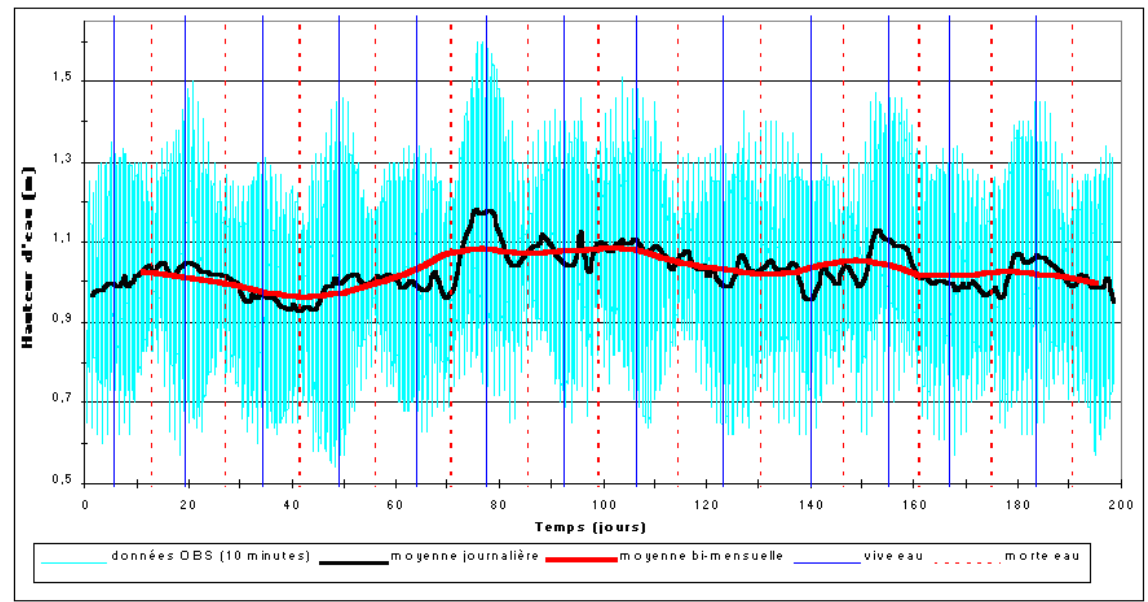

Figure 10. Evolution du niveau de l'eau sur 200 jours dans la lagune Ebrié.

\subsubsection{Salinité des eaux lagunaires}

La courbe présente une allure irrégulière (figure 11). Elle présente trois parties :

- De 0 à 65 jours, la courbe évolue dans les valeurs maximales de salinité (30,5 PSU). Elle croit progressivement pour atteindre la valeur maximale de 30,5 PSU au $22^{\text {ème }}$ puis décroît à 16 PSU au $28^{\text {ème }}$. Dans l'intervalle de 28 à 40 jours, la salinité augmente et diminue alternativement pour atteindre 10 PSU. Entre le 40 ème jour et $64^{\text {ème }}$ jour, elle oscille entre les valeurs de 17 à 25.5 PSU avant d'accéder à un taux relativement faible de 6 PSU au $64^{\text {ème }}$ jour.

- De 64 à 130 jours, l'allure de la courbe évolue dans les valeurs moyennes de la salinité. Elle oscille entre 5 et 16 PSU avant de baisser à 6 PSU au $130^{\text {ème jour. Dans }}$ cette deuxième partie le taux maximal de la salinité atteint est de 16 PSU et le minimum est de 5 PSU. 
- De 130 à 200 jours, l'allure de la courbe évolue dans les plus faibles taux de salinité. Elle varie entre 2 PSU, le taux minimum et 6 PSU, taux maximal.

Les variations de salinité en lagune se présentent en "marche d'escalier". Ces paliers coïncident avec les cycles saisonniers de l'arrière pays continental, de la lagune Ebrié et du domaine marin ivoirien. Le premier palier (février-avril) est le plus élevé. La forte salinité est due à l'absence des précipitations (saison sèche), aux faibles apports fluviaux liés à l'étiage du Comoé et surtout aux apports marins (saison marine à forte salinité). La seconde "marche d'escalier" s’observe d’avril à août. La salinité est faible car non seulement les précipitations sont abondantes mais aussi, c'est la période de crue de l'Agnéby et de La Mé. La fin de ce dernier palier correspond aux dernières grandes précipitations et surtout à la crue du Comoé (MONDE, 2004 ; GIRARD et al., 1971 ; BRENON et al., 2004) d'où l'observation d'une salinité encore plus faible. Ces résultats confirment l'impact des précipitations et des apports fluviaux (crue et étiage des fleuves et des rivières forestières) sur la salinité des eaux de la lagune. En effet, les fortes précipitations et les crues des fleuves réduisent le taux de salinité de par leurs apports (ruissellements continentaux, forts débits fluviaux, etc.) en milieu lagunaire. Par contre, la rareté des précipitations et l'étiage des fleuves augmentent le taux de salinité.

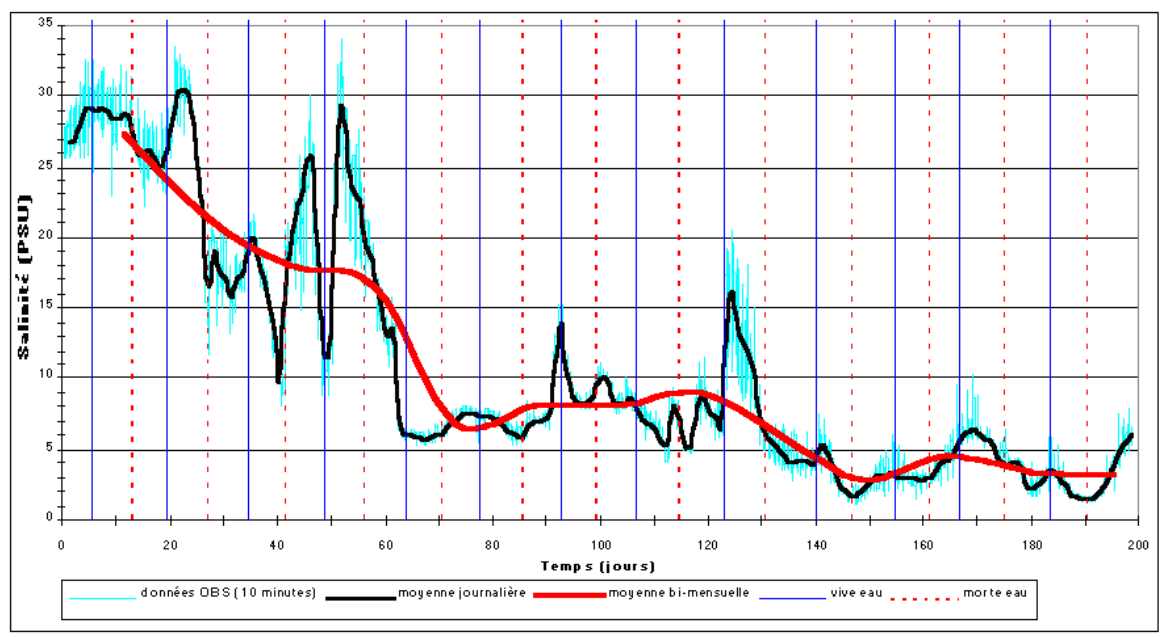

Figure 11. Salinité de la lagune Ebrié.

\subsection{3 Évolution de la température}

La figure 12 présente l'évolution de la moyenne journalière de températures en lagune Ebrié. L’allure de la courbe est généralement irrégulière. Elle est subdivisée en trois domaines :

- De 0 à 60 jours, l'allure de la courbe évolue dans un domaine de température moyenne. Elle vacille entre les valeurs de $27,5^{\circ} \mathrm{C}$ à des valeurs au dessus de $29,5^{\circ} \mathrm{C}$.

- De 60 à 140 jours la courbe atteint les valeurs maximales de la température au dessus de $30,5^{\circ} \mathrm{C}$. Dans l'intervalle de 60 à 125 jours, l'allure de la courbe croît et décroît 
respectivement et de façon alternative entre les températures au dessus de 28,5 et $30,5^{\circ} \mathrm{C}$. Au $125^{\text {ème }}$ jour, elle diminue jusqu'en dessous de $27,5^{\circ} \mathrm{C}$.

- De 140 à 200 jours, c'est le domaine de faible température. La courbe oscille entre 25,5 et $28,5^{\circ} \mathrm{C}$.

La température augmente durant la première phase où elle passe de 28 à $30,5^{\circ} \mathrm{C}$. Cette augmentation est due aux saisons sèches. Pendant ces périodes, les précipitations sont faibles et les apports du Comoé sont presque nuls (période d'étiage). Dans le second domaine, la courbe est décroissante. Cette baisse de température s'explique par les abondantes précipitations et par la crue de La Mé et de l’Agnéby. La dernière phase présente une évolution monotone. Les températures oscillent entre 26 et $27^{\circ} \mathrm{C}$. Ce palier s'étend de la mi-juillet à août. La baisse des températures est due aux fortes précipitations et à la crue du Comoé (MONDE, 2004).

Outre les apports fluviaux, la mer contribue à l'évolution des températures (DURAND \& GUIRAL, 1994 ; ELDIN, 1971 ; PAGES et al., 1979 ; ARFI et al., 1989 ; AFFIAN, 2003). En effet, à la mi-mars et à la mi-juin, les alizés du Sud-Ouest franchissent l'Équateur. Ils atteignent la Côte d'Ivoire et engendrent la mousson. Ces vents réguliers sont générateurs de remontées des eaux froides vers la surface ou upwellings. En juillet, la mousson remonte vers le Nord du pays : les pluies cessent et les vents se stabilisent ainsi que les upwellings. Pendant la période d'upwellings, une masse d'eau profonde se met en place. Au terme de cette période, les eaux guinéennes se répandent le long du littoral ivoirien qui se trouve ainsi baigné par des eaux chaudes de l'ordre de $28^{\circ} \mathrm{C}$ (MORLIERE \& REBERT, 1972).

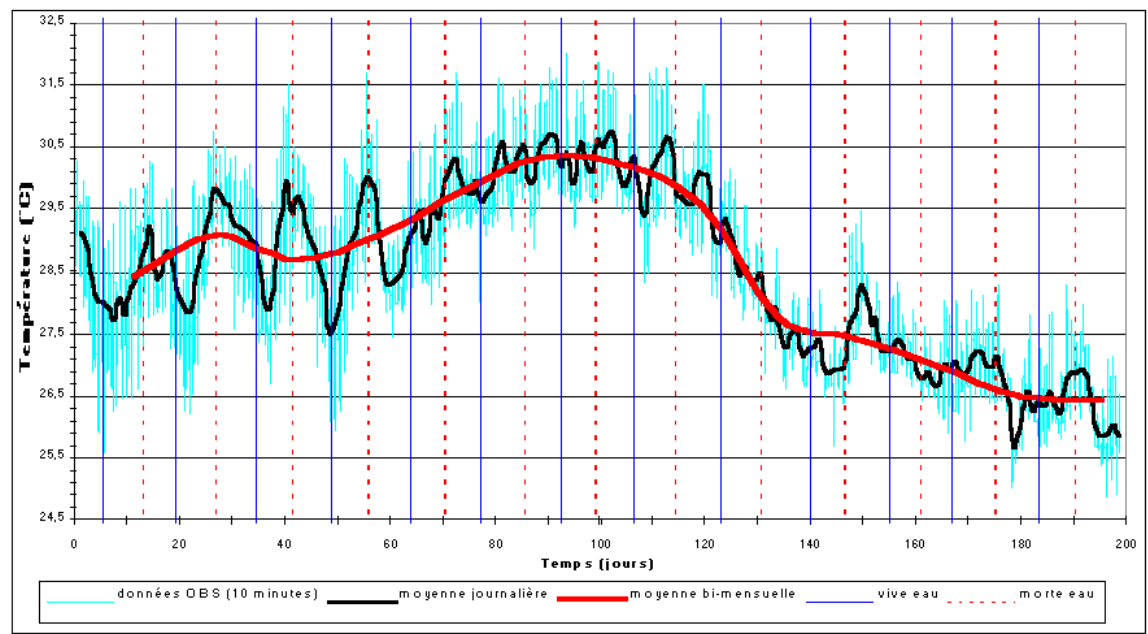

Figure 12. Température de la lagune Ebrié a différentes fréquences. 


\section{Conclusion}

La lagune Ebrié présente un chenal de -12 m avec de nombreuses morphostructures. Le dynamisme des baies estuariennes est très complexe. Dans un cycle de marée, le jusant prédomine sur le flot en durée et en intensité dans les baies, permettant ainsi le renouvellement des eaux lagunaires. Dans les baies estuariennes, les courants sont alternatifs dans les chenaux étroits et giratoires dans les zones étendues. Par exemple dans la baie de Cocody, les vitesses des courants sont proportionnelles à l'allure de l'onde de marée semi-diurne. Par contre dans la baie de Biétri, le caractère alternatif n’apparaît pas, du fait de son confinement et d’intenses aménagements industriels.

L'hydrodynamique dans les baies estuariennes montre que la variation de salinité s'agence dans les cycles saisonniers. Mais, les fortes précipitations et les crues des fleuves la réduisent. Les températures des eaux en lagune augmentent lors des faibles précipitations et de l'étiage du Comoé. La baisse de température est quant à elle, due aux abondantes précipitations et aux débits fluviaux.

\section{Références bibliographiques}

AFFIAN K. (2003). Approche environnementale d'un écosystème lagunaire microtidal (Lagune Ebrié, Côte d'Ivoire), par des études géochimiques, bathymétriques et hydrologiques: contribution du S.I.G. et de la télédétection. Thèse Doctorat, Univ. Cocody, 225 p.

ARFI R., GUIRAL D., TORRETON J. (1989). Cycle hydrologique annuel d'une baie eutrophe : La baie de Biétri (Lagune Ebrié, Côte d'Ivoire). Rev.Hydrobio. Trop., 22(4), pp 263-273.

BRENON I., MONDE S., POUVREAU N., MAURIN J.C. (2004). Modeling hydrodynamics in Ebrié lagoon (Côte d'Ivoire). Journal of African Earth Science, 39, pp 535-540. doi:10.1016/j.jafrearsci.2004.07.010

DURAND J.R., GUIRAL D. (1994). Hydroclimat et hydrochimie. In : Environnement et ressources aquatiques de Côte d'Ivoire. Les milieux lagunaires. T. 2 Edition Orstom, pp 59-90.

ELDIN M. (1971). Le climat. In : Le milieu naturel de la Côte d’Ivoire. Mém. Orstom, 50, pp 72-108.

GALLARDO Y. (1978). Assymetry and anomalies of circulation and vertical mixing in the branching of lagoon-estuary. Hydrodynamics of estuaries and fjords, Oceanography Ser., pp 197-206.

GIRARD G., SIRCOULON J., TOUCHEBOEUF P. (1971). Aperçu sur les régions hydrologiques. In: Le milieu naturel de la Côte d’Ivoire. Mém. Orstom, 50, pp 109-156.

GUIRAL D., LANUSSE A. (1984). Contribution à l'étude hydrodynamique de la baie de Biétri, lagune Ebrié, Côte d'Ivoire. Doc. Scientifique, CRO, Abidjan, 15(1-2), 1-18. 
LEMASSON L., PAGES J., DUFOUR P., CREMOUX J. (1981). Matière organique particulaire et biomasse dans une lagune tropicale. Rev. Hydrobio. Trop., 14(3), pp 191-212.

MONDE S. (2004). Etude et modélisation hydrodynamique de la circulation des masses d'eau dans la lagune Ebrié (Côte d'Ivoire). Thèse Doctorat, Université Cocody, 325 p.

MORLIERE A., REBERT J.P. (1972). Étude hydrologique du plateau continental ivoirien. Document Scientifique CRO, Abidjan, vol. 3, n², pp 1-30.

PAA -Port autonome d'Abidjan- (1999). Minutes de sondes de la zone portuaire. Carte bathymétrique de la baie d'Abidjan, n¹996-7-001, Échelle 1/1000, Rapport interne.

PAGES J., LEMASSON L., DUFOUR P. (1979). Éléments nutritifs et production primaire dans les lagunes de Côte d'Ivoire. Cycle annuel. Archive Scientifique du CRO, Abidjan, 5 (1), pp 1-60.

PERILLO G.M.E. (1995). Definitions and geomorphologic classifications of estuaries. Geomorphology and sedimentology of estuaries. Developments in Sedimentology, 53. Edited by G.M.E. Perillo, 17-47.

PRITCHARD D.W. (1955). Estuarine circulation patterns. Proceedings of the American Society of Civil Engineering. Vol. 81. pp 1-11.

TASTET J.P. (1979). Environnements sédimentaires et structuraux quaternaires du littoral du Golfe de Guinée (Côte d'Ivoire, Togo et Bénin). Thèse Doctorat, Univ. Bordeaux I, n 621, 175 p. 